УДК 531.39

\author{
РАСЧЕТ СОБСТВЕННЫХ КОЛЕБАНИЙ БУРИЛЬНОЙ \\ КОЛОННЫ С УЧЕТОМ ВЛИЯНИЯ ПРОМЫВОЧНОЙ ЖИДКОСТИ
}

\title{
INFLUENCE OF DRILLING FLUID ON FREE OSCILLATIONS \\ OF DRILLSTRING
}

Работа выполнена при поддержке РФФИ (грант № 17-01-00815а).

\author{
Погорелов Д.Ю. \\ Брянский государственный технический университет, \\ г. Брянск, Российская Федерация \\ D.Yu. Pogorelov \\ Bryansk State Technical University, \\ Bryansk, Russian Federation \\ e-mail: pogorelov@umlab.ru
}

Аннотация. Рассмотрен алгоритм расчета собственных колебаний бурильной колонны в скважине, ось которой задается произвольной пространственной кривой. Бурильная колонна моделируется геометрически нелинейными балочными конечными элементами с использованием модифицированного подхода Крейга-Бемптона. Уравнения движения колонны линеаризуются в окрестности положения равновесия.

Для учета влияния жидкости используется полученное авторами S.S. Chen, M.W. Wambsganss, J.A. Jendrzejczyk аналитическое выражение для коэффициентов вязкого сопротивления жидкости и присоединенной массы в случае плоской задачи о гармонических колебаниях кругового цилиндра в трубе, заполненной несжимаемой ньютоновской жидкостью. 
С учетом модели Чена матрицы масс и демпфирования линеаризованной модели зависят от частоты колебаний. В данной работе приведено решение Чена, анализируются алгоритмы расчета коэффициентов диссипации и присоединенных масс через модифицированные функции Бесселя в условиях плохой обусловленности выражений для этих коэффициентов. Предложен быстрый итерационный алгоритм расчета собственных частот и форм колебаний колонны, а также метод оценки степени демпфирования каждой частоты. Разработанные алгоритмы реализованы в программном комплексе «Универсальный механизм» (ПК УМ).

Abstract. Computation of natural frequencies and modes of a drillstring in a borehole with an arbitrary centerline is considered. The drill string is modeled by geometrically nonlinear beam elements using the modified Craig-Bampton method. Equations of motion are linearized near the equilibrium position.

Results received by S.S. Chen, M.W. Wambsganss, J.A. Jendrzejczyk are used for a fluid model. Analytic expressions for damping and added mass coefficients are used for plane vibrations of a cylindrical rod in incompressible Newtonian fluid enclosed by a concentric cylindrical shell (the Chen solution).

Taking into account the Chen model, mass and damping matrices of the linearized model depend on the vibration frequency. In this paper we consider the Chen solution, analyze algorithms for computation of damping and added mass coefficients provided the ill-conditioning of expressions for the coefficients in modified Bessel functions. A fast algorithm for computation of natural frequencies and modes as well as a method for evaluation of damping ratio for each of the frequency are suggested. The discussed algorithms are implemented in the Universal Mechanism software.

Ключевые слова: динамика бурильной колонны, учет влияния промывочной жидкости, спектральный анализ.

Key words: drillstring dynamics, influence of drilling fluid, spectral analysis. 


\section{Введение}

Бурильная колонна моделируется геометрически нелинейными балочными конечными элементами с использованием модифицированного подхода Крейга-Бемптона $[1,2]$. Уравнения движения колонны линеаризуются в окрестности положения равновесия.

Для учета влияния жидкости используются результаты работы [3], в которой получено аналитическое выражение для коэффициентов вязкого сопротивления жидкости и присоединенной массы в случае плоской задачи о гармонических колебаниях кругового цилиндра в трубе, заполненной несжимаемой ньютоновской жидкостью (в дальнейшем будем называть его решением Чена).

В данной работе приведено решение Чена, анализируются алгоритмы расчета коэффициентов диссипации и присоединенных масс через модифицированные функции Бесселя в условиях плохой обусловленности выражений для этих коэффициентов. Предложен быстрый итерационный алгоритм расчета собственных частот и форм колебаний колонны, а также метод оценки степени демпфирования каждой частоты. Разработанные алгоритмы реализованы в программном комплексе «Универсальный механизм» (ПК УМ) $[4,5]$.

Компьютерное моделирование динамики процессов бурения является актуальной проблемой механики и имеет большое прикладное значение, в частности, для расчета напряженно-деформированного состояния бурильной колонны [6-10]. Одной из наиболее сложных проблем при моделировании является учет взаимодействия колонны с промывочной жидкостью. Использование трехмерных гидродинамических моделей в силу большой длины скважины является слишком трудоемким с вычислительной точки зрения и не может использоваться для оперативных расчетов, поэтому на практике реализуются приближенные модели. Как правило, воздействие 
жидкости на поперечные колебания бурильной колонны учитывается с помощью сил вязкого сопротивления и понятия присоединенной массы.

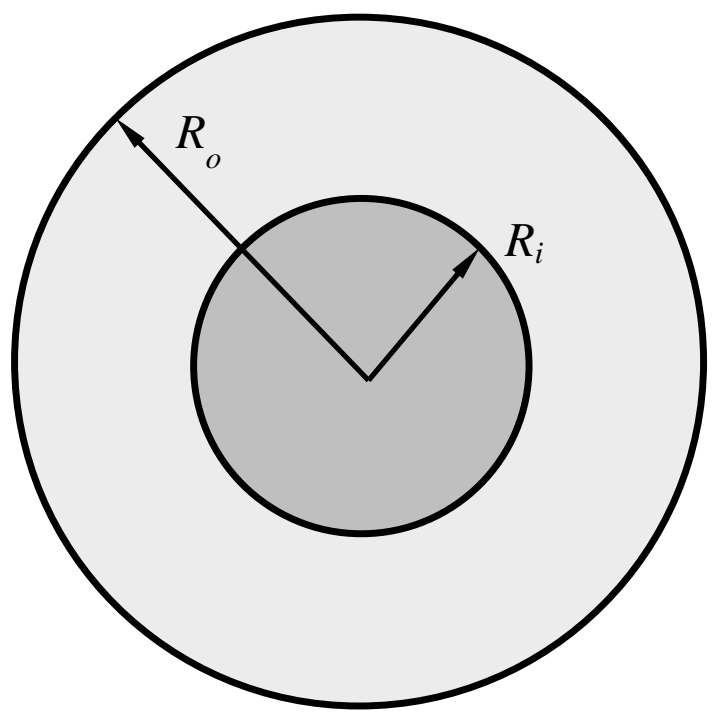

Рисунок 1. Цилиндр в трубе, заполненной жидкостью

В настоящее время отсутствуют универсальные и точные модели сил сопротивления и присоединенных масс, учитывающие эксцентриситет в положении колонны относительно скважины, наличие продольного потока жидкости, ее неньютоновские характеристики и другие эффекты, усложняющие модели. Вместе с тем, при решении некоторых частных динамических проблем могут быть использованы известные аналитические решения. Рассмотрим проблему спектрального анализа уравнений движения бурильной колонны, линеаризованных относительно положения равновесия в скважине с криволинейной осевой линией. Поскольку в соответствии с решением Чена [3] коэффициент сопротивления и присоединенная масса зависят от частоты возмущения, этим свойством обладают матрицы масс и демпфирования линеаризованных уравнений, что значительно осложняет расчет собственных частот колебаний бурильной колонны.

\section{Решение Чена для колебаний цилиндра в трубе}

В статье [3] получено полезное решение, связанное с малыми гармоническими колебаниями кругового цилиндра, заполненной несжимаемой вяз- 
кой жидкостью (рисунок 1). Задача решается в плоской постановке. Центр радиальных колебаний цилиндра совпадает с центром трубы. Результатом является выражение для силы, приходящейся на единицу длины цилиндра и действующей со стороны жидкости на цилиндр. Выражение имеет два слагаемых

$$
F=C_{m} \rho A \frac{\mathrm{d}^{2} r}{\mathrm{~d} t^{2}}-C_{d} \omega \rho A \frac{\mathrm{d} r}{\mathrm{~d} t},
$$

где $r$ - радиальное смещение цилиндра при колебаниях;

$\omega$ - частота колебаний, рад/с;

$A$ - площадь кольцевого зазора;

$\rho$ - плотность жидкости.

Первое слагаемое в выражении (1) зависит от ускорения и соответствует присоединенной массе жидкости, второе слагаемое зависит от скорости цилиндра и определяет диссипативные свойства жидкости.

Коэффициенты присоединенной массы и демпфирования $C_{m}(\omega), C_{d}(\omega)$ зависят от частоты колебаний $\omega$, радиусов $R_{i}, R_{O}$ и кинематической вязкости жидкости $v$ через параметры

$$
\alpha=\lambda R_{i}, \beta=\lambda R_{o}, \gamma=R_{i} / R_{o}, \lambda=\sqrt{i \frac{\omega}{v}} .
$$

Здесь $i$ - мнимая единица.

Коэффициенты являются действительной и мнимой частями комплекснозначной функции $H[3,11]$

$$
\begin{gathered}
C_{m}=\operatorname{Re} H, C_{d}=-\operatorname{Im} H, \\
H=\frac{H_{c}}{H_{d}}-1, \\
H_{c}=\alpha^{2}\left(1-\gamma^{2}\right)\left[I_{0}(\alpha) K_{0}(\beta)-I_{0}(\beta) K_{0}(\alpha)\right]+\alpha\left[I_{1}(\alpha) K_{0}(\beta)+I_{0}(\beta) K_{1}(\alpha)\right]+ \\
4 \alpha \gamma\left[I_{0}(\alpha) K_{1}(\beta)+I_{1}(\beta) K_{0}(\alpha)\right]-8 \gamma\left[I_{1}(\alpha) K_{1}(\beta)-I_{1}(\beta) K_{1}(\alpha)\right],
\end{gathered}
$$




$$
\begin{gathered}
H_{d}=2 \alpha^{2}\left[I_{0}(\alpha) K_{0}(\beta)-I_{0}(\beta) K_{0}(\alpha)\right]+2 \alpha \gamma\left[I_{0}(\alpha) K_{1}(\beta)-I_{1}(\beta) K_{0}(\beta)+\right. \\
\left.+I_{1}(\beta) K_{0}(\alpha)-I_{0}(\beta) K_{1}(\beta)\right]+2 \alpha \gamma^{2}\left[I_{0}(\beta) K_{1}(\alpha)+I_{0}(\alpha) K_{1}(\alpha)+I_{1}(\alpha) K_{0}(\beta)-\right. \\
\left.-I_{1}(\alpha) K_{0}(\alpha)\right] .
\end{gathered}
$$

В этих выражениях $I_{0}, I_{1}, K_{0}, K_{1}$ - модифицированные функции Бесселя первого и второго рода, для расчета которых используются их выражения через функции Кельвина

$$
i^{n} I_{n}(x \sqrt{i})=\operatorname{ber}_{n} x+i \operatorname{bei}_{n} x, i^{-n} K_{n}(x \sqrt{i})=\operatorname{ker}_{n} x+i \operatorname{kei}_{n} x
$$

Практический расчет численных значений коэффициентов (2) во всем диапазоне изменения параметров модели осложняется в силу плохой обусловленности приведенных выражений (3). Полезным является асимптотическое приближение для функции $H$, справедливое при больших значениях параметров $\alpha, \beta$ и при узком кольцевом зазоре, т.е. $\gamma \approx 1$ [3]:

$$
\begin{aligned}
& H=\frac{\left[\alpha^{2}\left(1+\gamma^{2}\right)-8 \gamma\right] s_{h}+2 \alpha\left(2-\gamma+\gamma^{2}\right) c_{h}-2 \gamma^{2} \sqrt{\alpha \beta}-2 \alpha \gamma \sqrt{\gamma}}{\alpha^{2}\left(1-\gamma^{2}\right) s_{h}-2 \alpha \gamma(1+\gamma) c_{h}+2 \gamma^{2} \sqrt{\alpha \beta}+2 \alpha \gamma \sqrt{\gamma}}, \\
& s_{h}=\sinh (\beta-\alpha), c_{h}=\cosh (\beta-\alpha) .
\end{aligned}
$$

Вместе с тем, вне указанного диапазона изменения параметров, в частности, при малых частотах возмущения, асимптотическое приближение (4) имеет большую погрешность и следует использовать точные формулы (3). Для численной стабилизации этих выражений предлагается использовать асимптотические свойства модифицированных функций Кельвина, выполнив преобразование

$$
I_{n}(x \sqrt{i})=I_{n}^{\prime}(x \sqrt{i}) e^{x / \sqrt{2}}, K_{n}(x \sqrt{i})=K_{n}^{\prime}(x \sqrt{i}) e^{-x / \sqrt{2}} .
$$

В отличие от исходных функций, нормированные функции $I_{n}^{\prime}, K_{n}^{\prime}$ при росте аргумента $x$ стремятся к конечным значениям.

В нормированных функциях Бесселя выражения (3) обладают значительно большей устойчивостью к ошибкам вычисления 


$$
\begin{gathered}
H_{c}=\alpha^{2}\left(1-\gamma^{2}\right)\left[\kappa I_{0}^{\prime}(\alpha) K_{0}^{\prime}(\beta)-I_{0}^{\prime}(\beta) K_{0}^{\prime}(\alpha) / \kappa\right]+\alpha\left[\kappa I_{1}(\alpha) K_{0}(\beta)+\right. \\
\left.+I_{0}(\beta) K_{1}(\alpha) / \kappa\right]+4 \alpha \gamma\left[\kappa I_{0}(\alpha) K_{1}(\beta)+I_{1}(\beta) K_{0}(\alpha) / \kappa\right]-8 \gamma\left[\kappa I_{1}(\alpha) K_{1}(\beta)-\right. \\
\left.-I_{1}(\beta) K_{1}(\alpha) / \kappa\right] \\
H_{d}=2 \alpha^{2}\left[\kappa I_{0}(\alpha) K_{0}(\beta)-I_{0}(\beta) K_{0}(\alpha) / \kappa\right]+2 \alpha \gamma\left[\kappa I_{0}(\alpha) K_{1}(\beta)-I_{1}(\beta) K_{0}(\beta)+\right. \\
\left.+I_{1}(\beta) K_{0}(\alpha) / \kappa-I_{0}(\beta) K_{1}(\beta)\right]+2 \alpha \gamma^{2}\left[I_{0}(\beta) K_{1}(\alpha) / \kappa+I_{0}(\alpha) K_{1}(\alpha)+\kappa I_{1}(\alpha) K_{0}(\beta)-\right. \\
\left.-I_{1}(\alpha) K_{0}(\alpha)\right] \\
\kappa=e^{\sqrt{\frac{\omega}{2 v}}\left(R_{i}-R_{o}\right)}
\end{gathered}
$$

Для вычисления функций Кельвина используются процедуры, опубликованные на сайте [12]. Расчеты производятся с двойной точность.

На рисунке 2 приведено сравнение точного (5) и асимптотического (4) решений для коэффициентов присодиненной массы и демпфирования (2) в зависимости от частоты колебаний. Расчеты выполнены при следующих значения параметров:

$$
\rho=1200 \kappa \Gamma / \mathrm{M}^{3}, R_{i}=95 \mathrm{Mм}, R_{O}=105 \mathrm{Mм}, \mu=\rho \mathrm{v}=0.015 \text { Па } \cdot \mathrm{c} .
$$
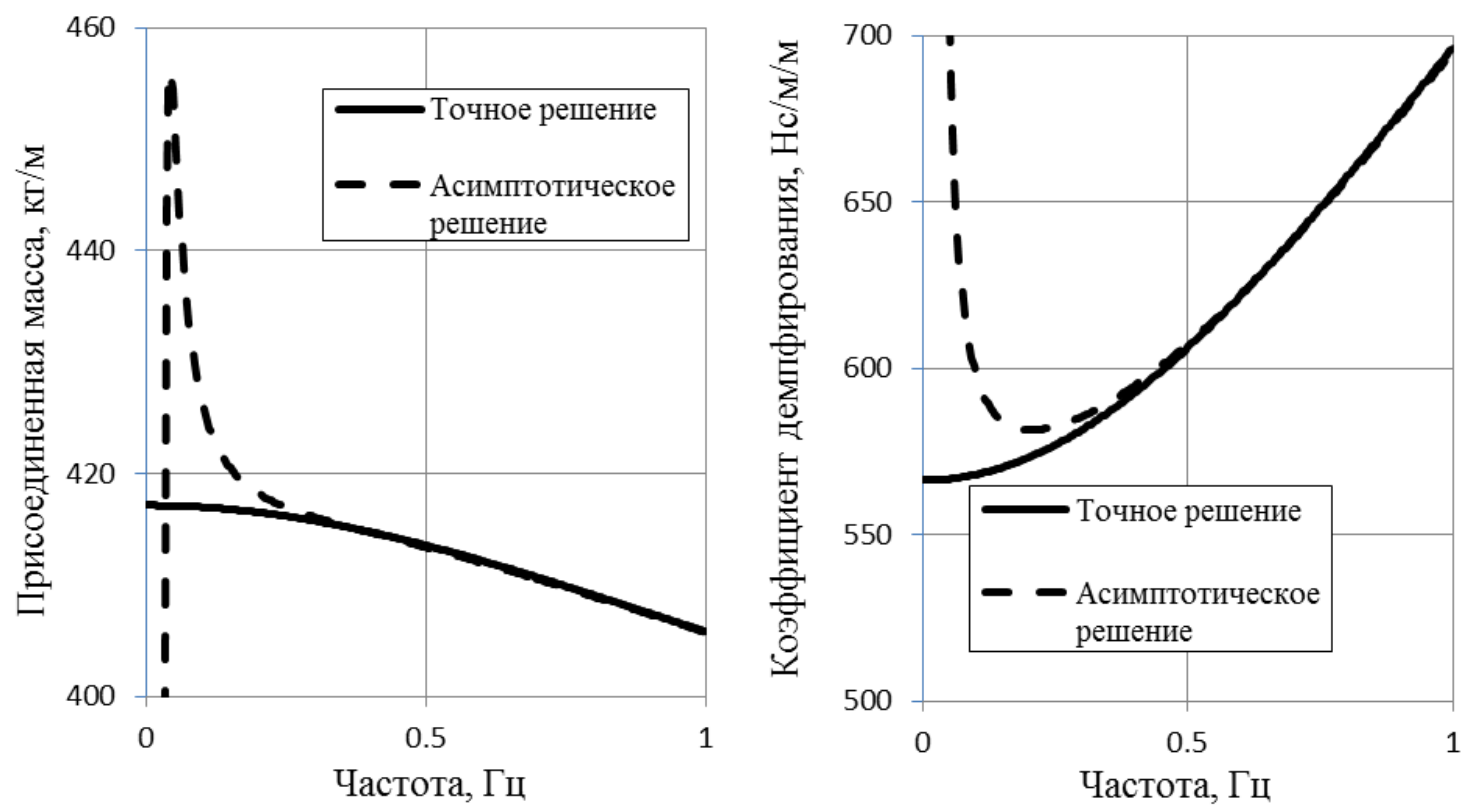

Рисунок 2. Сравнение точного и асимптотического решений

Таким образом, при малых значениях частоты следует использовать точное решение. Асимптотическое решение должно использоваться при 
достаточно больших частотах, при которых расчет по формулам (5) становится невозможным.

Влияние радиуса трубы $R_{i}$ на значения рассчитываемых коэффициентов при тех же параметрах показано на рисунке 3. Характерным является быстрый рост коэффициентов с уменьшением зазора между трубой и стенками скважины, т.е. при $R_{i} \rightarrow R_{o}[3,11]$.
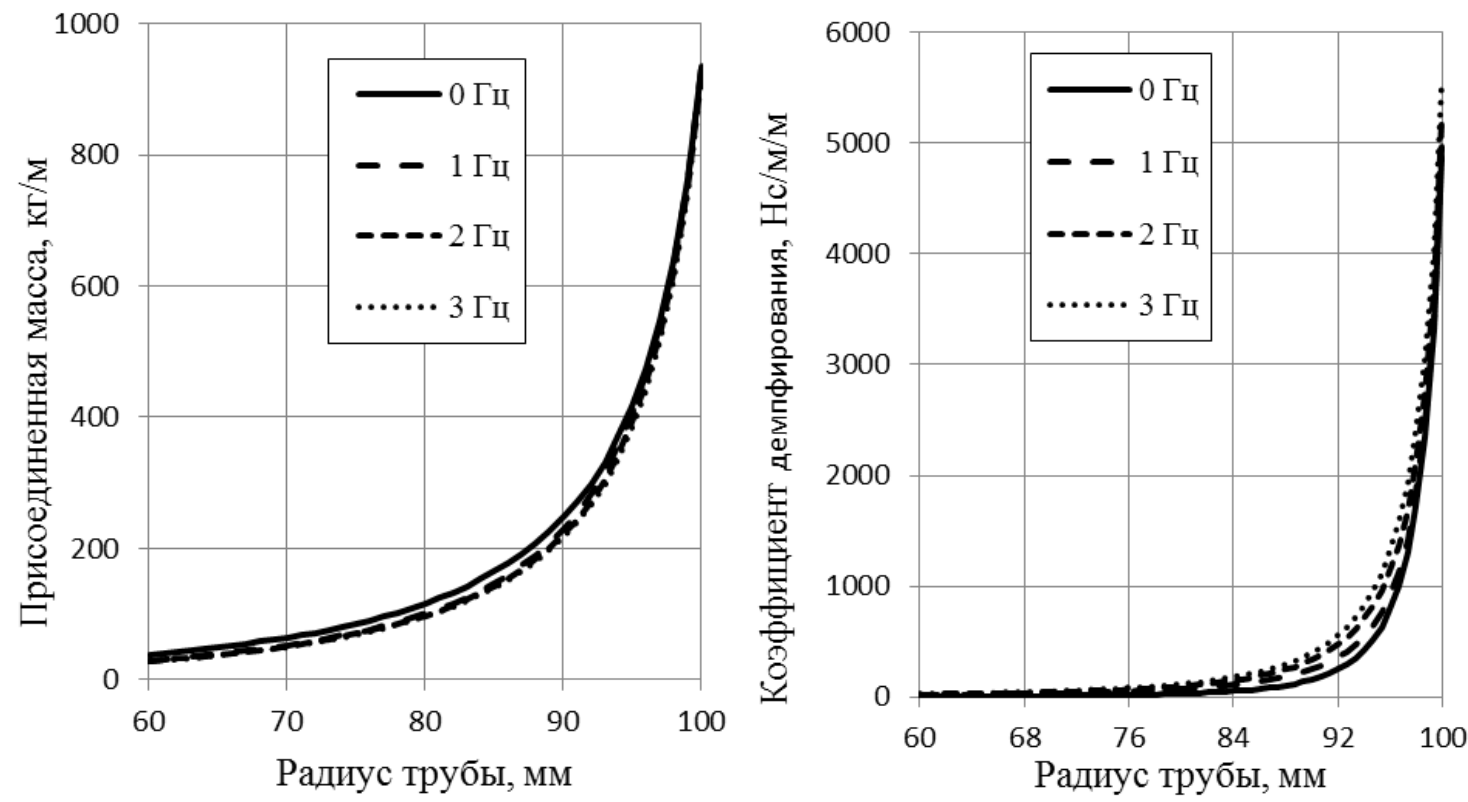

Рисунок 3. Зависимость коэффициентов присоединенной массы и демпфирования от радиуса трубы

\section{Спектральный анализ бурильной колонны}

Рассмотрим сначала задачу расчета собственных частот и форм колебаний бурильной колонны в скважине с учетом влияния жидкости. Поскольку присоединенная масса жидкости зависит от частоты колебаний, корректная формулировка проблемы выглядит следующим образом:

$$
.\left(M(\omega) \omega^{2}-C\right) e=0 .
$$

Здесь $M(\omega), C$ - матрицы масс и коэффициентов жесткости линеаризованных уравнений движения;

$e$ - собственный вектор, определяющий форму колебаний с частотой $\omega$. 
Поскольку уравнения линеаризуются в окрестности устойчивого положения равновесия бурильной колонны, то обе матрицы являются положительно определенными.

С точки зрения практической реализации, наиболее простым методом расчета спектра задачи (6), является прием, основанный на многократном решении стандартной задачи

$$
\left(M\left(\omega^{*}\right) \omega^{2}-C\right) e=0
$$

для фиксированных значений частоты $\omega^{*}=\omega^{*}{ }_{1}<\omega^{*}{ }_{2} \ldots<\omega^{*}{ }_{N}$.

Рассмотрим пример такого подхода для компоновки низа буровой колонны, находящейся в положении равновесия в скважине с криволинейной осевой линией, рисунок 4. Модель содержит 156 балочных конечных элементов и имеет 942 степени свободы.

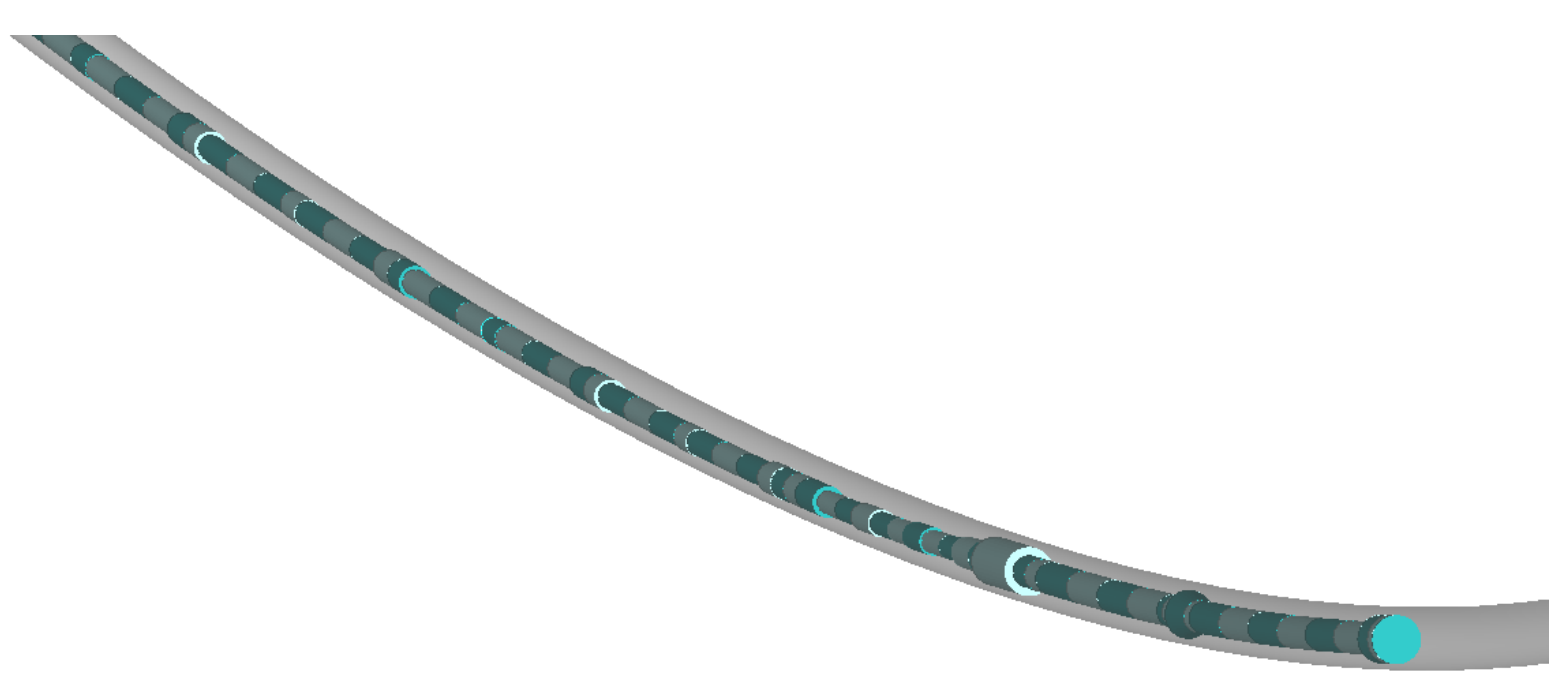

Рисунок 4. Модель компоновки низа бурильной колонны в криволинейной скважине

Расчет собственных частот линеаризованных уравнений движения бурильной колонны при различных значениях частоты $\omega^{*}$ выполнен в ПК УМ с использованием QR алгоритма. Результаты для низших частот показаны на рисунке 5. Решению спектральной задачи (6) соответствуют точки пересечения графика для каждой из частот с биссектрисой, указанной на рисунке пунктирной линией; четыре таких решения отмечены кружками. 


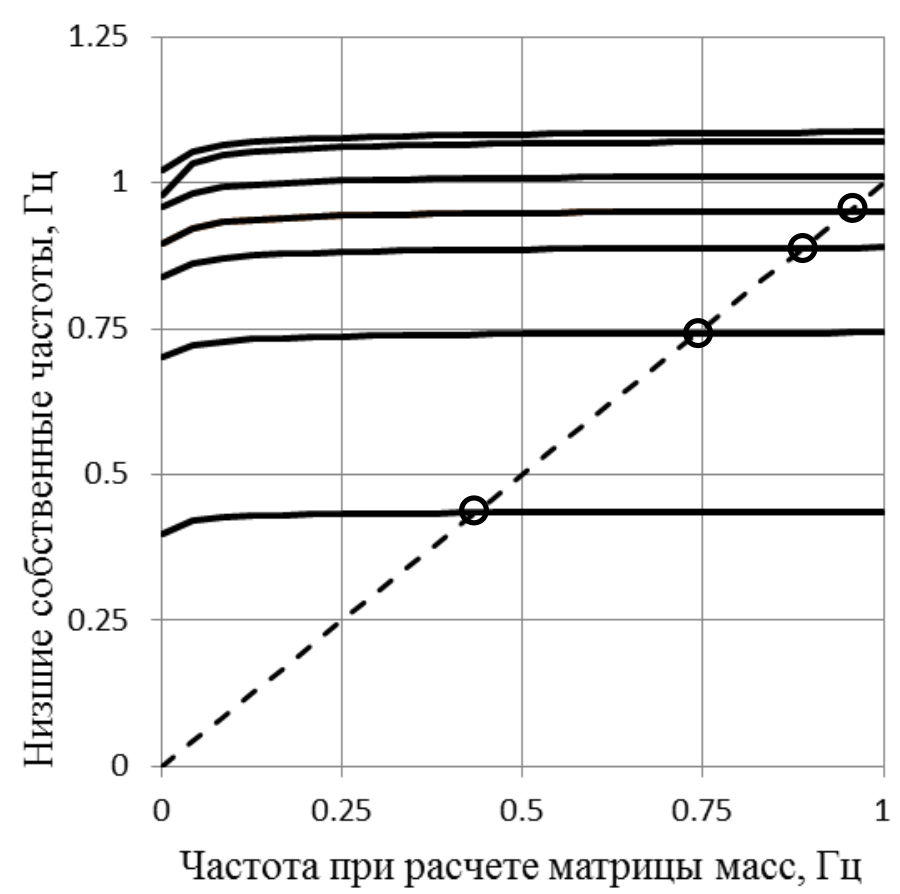

Рисунок 5. Зависимость низших собственных частот от $\omega^{*}$

Приведенный метод решения спектральной задачи (6) имеет значительную вычислительную трудоемкость. Рассмотрим альтернативный, гораздо более эффективный итерационный алгоритм, который, наряду с собственными частотами, позволяет определить собственные формы колебаний. Выберем некоторое опорное значение частоты $\omega^{*}$, например, $\omega^{*}=1$ Гц и определим численное решение задачи (7)

$$
\begin{aligned}
& \left(M\left(\omega^{*}\right) \omega_{i, 0}^{2}-C\right) e_{i, 0}=0, i=1,2, \ldots, n \\
& \omega_{1,0} \leq \omega_{2,0} \leq \ldots \leq \omega_{n, 0} .
\end{aligned}
$$

Используем это решение в качестве нулевого приближения для поиска решений задачи (6) с использованием метода обратных итераций [13].

Алгоритм ищет решения проблемы (6) в цикле по частотам $\omega_{i, 0}, i=1,2 \ldots$ Далее при описании алгоритма опустим индекс частоты $i$.

Шаг 1. Итерационная оценка значения частоты для заданного собственного вектора 


$$
\omega_{k}^{2}=\frac{e_{0}^{T} C e_{0}}{e_{0}^{T} M\left(\omega_{k-1}\right) e_{0}}, k=1,2, \ldots
$$

Шаг заканчивается при выполнении условия $\left|\omega_{m}-\omega_{m-1}\right|<\varepsilon_{1}$, где $\varepsilon_{1}$ - малое число.

Результат $\omega^{*}=\omega_{m}$ вместе с матрицей-столбцом $e^{0}=e_{0}$ является начальным приближением для итераций второго шага.

Шаг 2. Обратные итерации для определения решения задачи (7) с матрицей масс, вычисленной для $\omega^{*}$

$$
\begin{aligned}
& \left(M\left(\omega^{*}\right)\left(\omega^{*}\right)^{2}-C\right) \tilde{e}^{k}=e^{k-1}, \quad k=1,2 \ldots \\
& e^{k}=\tilde{e}^{k} /\left\|\tilde{e}^{k}\right\|_{\infty}, \Delta\left(\omega^{k}\right)^{2}=1 /\left\|\tilde{e}^{k}\right\|_{\infty} .
\end{aligned}
$$

Обратные итерации прерываются при выполнении условия $\left\|e^{k}-e^{k-1}\right\|<\varepsilon_{e}$, где $\varepsilon_{e}-$ малое число. Новое значение квадрата частоты $\omega^{2}=\left(\omega^{*}\right)^{2}+\Delta\left(\omega^{k}\right)^{2}$.

Если найденная поправка к квадрату частоты достаточно мала $\Delta\left(\omega^{k}\right)^{2} / \omega^{2}<\varepsilon$, то найденная частоты и собственный вектор $e^{k}$ принимаются в качестве решения задачи (6), в противном случае идет переход к шагу 1 с новыми значениями частоты и вектора.

Описанный двухуровневый итерационный алгоритм позволяет ограничиться расчетом нужного числа частот спектра и сравнительно быстро определить как собственные частоты, так и собственные векторы задачи (6). В таблице 1 приведено сравнение данного решения, обозначенного как «точное», с решениями задачи (7) для семи низших частот. В качестве опорных частот $\omega^{*}$ задачи (7) задавалось значения «точного» решения. Первая строка в таблице содержит «точное» решение, а остальные - частоты спектра задачи (7) при опорных частотах, равных первой, второй, третьей и седьмой частотам «точного» решения (6). При таком задании опорной частоты спектр решения задачи (7) должен содержать как мини- 
мум одну частоту, значение которой равно или близко к опорной частоте; соответствующие числа в таблице 1 выделены жирным шрифтом и подтверждают корректность расчетов «точного» решения.

Таблица 1.Сравнение точного решения с расчетами при фиксированных значениях частоты $\omega^{*}$, Гц

\begin{tabular}{|l|c|c|c|c|c|c|c|}
\hline & 1 & 2 & 3 & 4 & 5 & 6 & 7 \\
\hline Точное решение & 0,434384 & 0,742049 & 0,888217 & 0,951085 & 1,01143 & 1,07144 & 1,08695 \\
\hline$\omega^{*}=0,434384$ & $\mathbf{0 , 4 3 4 3 8 4}$ & 0,739807 & 0,884767 & 0,94707 & 1,00708 & 1,06585 & 1,08171 \\
\hline$\omega^{*}=0,742049$ & 0,435881 & $\mathbf{0 , 7 4 2 0 4 9}$ & 0,887457 & 0,949972 & 1,01004 & 1,06945 & 1,08505 \\
\hline$\omega^{*}=0,888217$ & 0,436303 & 0,742683 & $\mathbf{0 , 8 8 8 2 1 7}$ & 0,950791 & 1,01087 & 1,07047 & 1,08599 \\
\hline$\omega^{*}=1,08695$ & 0,436736 & 0,743332 & 0,888995 & 0,951631 & 1,01173 & 1,07152 & $\mathbf{1 , 0 8 6 9 6}$ \\
\hline
\end{tabular}

В заключение укажем метод приближенной оценки степени демпфирования каждой из частот бурильной колонны с учетом влияния жидкости на коэффициент демпфирования. Линейные уравнения свободных колебаний в окрестности устойчивого положения равновесия имеют вид

$$
M(\omega) \frac{d^{2} x}{d t^{2}}+D(\omega) \frac{d x}{d t}+C x=0
$$

Здесь $D(\omega)$ - симметричная матрица демпфирования, зависящая от частоты возмущения, $x$ - матрица-столбец обобщенных координат модели. Поскольку демпфирование, вызванное жидкостью, обычно является малым, степень демпфирования можно оценить по приближенному уравнению, которое можно получить по аналогии с переходом к главным координатам:

$$
\begin{aligned}
& m_{i} \frac{d^{2} q_{i}}{d t^{2}}+d_{i} \frac{d q_{i}}{d t}+c_{i} q_{i}=0, i=1,2, \ldots, n \\
& m_{i}=e_{i}^{T} M\left(\omega_{i}\right) e_{i}, d_{i}=e_{i}^{T} D\left(\omega_{i}\right) e_{i}, c_{i}=e_{i}^{T} C e_{i}=\omega_{i}^{2} m_{i}
\end{aligned}
$$

Здесь $\omega_{i}, e_{i}$ - решение спектральной задачи (6). В этом уравнении мы пренебрегаем связью между собственными формами колебаний через мат- 
рицу демпфирования. В результате получим оценку доли демпфирования для частоты $\omega_{i}$

$$
\beta_{i} \approx \frac{d_{i}}{2 m_{i} \omega_{i}}
$$

\section{Выводы}

1. Предложенный метод, состоящий в комбинации точного и асимптотического решений, позволяет рассчитывать коэффициенты присоединенной массы и демпфирования в зависимости от частоты возмущения во всем диапазоне параметров задачи.

2. Для расчета собственных частот форм колебаний бурильной колонный в скважине предложен подход, в основе которого лежит метод обратных итераций. Результаты демонстрируют корректность и точность представленного метода.

\section{Список используемых источников}

1. Pogorelov D., Mikheev G., Lysikov N., Ring L., Gandikota R., Abedrabbo N. A Multibody System Approach to Drill String Dynamics Modeling // Proc. ASME 11th Biennial Conference on Engineering Systems Design and Analysis (ESDA2012). 2012. Vol. 4. Pp. 53-62. DOI: 10.1115/ESDA2012-82316.

2. Dmitrochenko O., Mikheev G., Pogorelov D., Gandikota R. A Nonlinear Finite Element for Simulation of Dynamics of Beam Structures Using Multibody System Approach // Proc. 11th World Congress on Computational Mechanics (WCCM XI). 2014. P. 655-666.

3. Chen S.S., Wambsganss M.W., Jendrzejczyk J.A. Added Mass and Damping of a Vibrating Rod in Confined Viscous Fluids // Journal of Applied Mechanics. 1976. Vol. 43. P. 325-329. DOI:10.1115/1.3423833. 
4. Погорелов Д.Ю. Компьютерное моделирование динамики технических систем с использованием программного комплекса «Универсальный механизм» // Вестник компьютерных и информационных технологий. 2005. № 4. С. 27-34.

5. Сайт «Универсальный механизм» [Электронный ресурс]. Режим доступа: http://www.universalmechanism.com (дата обращения: 14.05.2018).

6. Christoforou A.P., Yigit A.S. Fully Coupled Vibrations of Actively Controlled Drillstrings // Journal of Sound and Vibrations. 2003. Vol. 257. No. 5. P. 1029-1045. DOI: 10.1016/S0022-460X(03)00359-6.

7. Sampaio R., Piovan M.T., Venero Lozano G. Coupled Axial/Torsional Vibrations of Drill-Strings by Means of Non-Linear Model // Mechanics Research Communications. 2007. Vol. 34. No. 5-6. P. 497-502. DOI: 10.1016/j.mechrescom.2007.03.005.

8. Khulief Y.A., Al-Sulaiman F.A., Bashmal S. Vibration Analysis of Drillstrings with Self-Excited Stick-Slip Oscillations // Journal of Sound and Vibration. 2007. Vol. 299. P. 540-558. DOI: 10.1016/j.jsv.2006.06.065.

9. Ren F.S., Chen S., Yao Z.G. Dynamics Analysis and Vibration Suppression of a Flexible Rotation Beam // Applied Mechanics and Materials. 2012. Vol. 214. P. 165-172. DOI: https://doi.org/10.4028/www.scientific.net/ AMM.214.165.

10. Ritto T.G., Sampaio R., Soize C. Drill-String Dynamics Coupled with the Drilling Fluid Dynamics // Proc. XIII International Symposium on Dynamic Problems of Mechanics (DINAME 2009). Rio de Janeiro, 2009. P. 1-10. URL: https://hal.archives-ouvertes.fr/hal-00691722.

11. Paidoussis M.P. Fluid-Structure Interactions: Slender Structures and Axial Flow. London: Academic Press, 1998. Vol. 1. 573 p.

12. Цилиндрические функции [Электронный ресурс]. Режим доступа: http://num-anal.srcc.msu.ru/lib_na/cat/cat64.htm (дата обращения: 14.05.2018). 
13. Амосов А.А., Дубинский Ю.А., Копченова Н.В. Вычислительные методы для инженеров. М.: Высш. шк., 1994. 544 с.

\section{References}

1. Pogorelov D., Mikheev G., Lysikov N., Ring L., Gandikota R., Abedrabbo N. A Multibody System Approach to Drill String Dynamics Modeling. Proc. ASME 11th Biennial Conference on Engineering Systems Design and Analysis (ESDA2012), 2012, Vol. 4, pp. 53-62. DOI: 10.1115/ESDA2012-82316.

2. Dmitrochenko O., Mikheev G., Pogorelov D., Gandikota R. A Nonlinear Fi-nite Element for Simulation of Dynamics of Beam Structures Using Multibody System Approach. Proc. 11th World Congress on Computational Mechanics (WCCM XI), 2014, pp. 655-666.

3. Chen S.S., Wambsganss M.W., Jendrzejczyk J.A. Added Mass and Damping of a Vibrating Rod in Confined Viscous Fluids. Journal of Applied Mechanics, 1976, Vol. 43, pp. 325-329. DOI:10.1115/1.3423833.

4. Pogorelov D.Yu. Komp'yuternoe modelirovanie dinamiki tekhnicheskikh sistem $\mathrm{s}$ ispol'zovaniem programmnogo kompleksa «Universal'nyi mekhanizm» [Computer simulation of technical system dynamics with Universal Mechanism Software]. Vestnik komp'yuternykh $i$ informatsionnykh tekhnologii - Herald of Computer and Information Technologies, 2005, No. 4, pp. 27-34. [in Russian].

5. Sait «Universal'nyi mekhanizm» [Universal Mechanism Website] [Electronic Resource]. Available at: http://www.universalmechanism.com (accessed 14.05.2018). [in Russian].

6. Christoforou A.P., Yigit A.S. Fully Coupled Vibrations of Actively Controlled Drillstrings. Journal of Sound and Vibrations, 2003, Vol. 257, No. 5, pp. 1029-1045. DOI: 10.1016/S0022-460X(03)00359-6. 
7. Sampaio R., Piovan M.T., Venero Lozano G. Coupled Axial/Torsional Vibrations of Drill-Strings by Means of Non-Linear Model. Mechanics Research Communications, 2007, Vol. 34, No. 5-6, pp. 497-502. DOI: 10.1016/j.mechrescom.2007.03.005.

8. Khulief Y.A., Al-Sulaiman F.A., Bashmal S. Vibration Analysis of Drillstrings with Self-Excited Stick-Slip Oscillations. Journal of Sound and Vibration, 2007, Vol. 299, pp. 540-558. DOI: 10.1016/j.jsv.2006.06.065.

9. Ren F.S., Chen S., Yao Z.G. Dynamics Analysis and Vibration Suppression of a Flexible Rotation Beam. Applied Mechanics and Materials, 2012, Vol. 214, pp. 165-172. DOI: https://doi.org/10.4028/www.scientific.net/ AMM.214.165.

10. Ritto T.G., Sampaio R., Soize C. Drill-String Dynamics Coupled with the Drilling Fluid Dynamics. Proc. XIII International Symposium on Dynamic Prob-lems of Mechanics (DINAME 2009). Rio de Janeiro, 2009. P. 1-10. URL: https://hal.archives-ouvertes.fr/hal-00691722.

11. Paidoussis M.P. Fluid-Structure Interactions: Slender Structures and Axial Flow. London: Academic Press, 1998. Vol. 1. 573 p.

12. Tsilindricheskie funktsii [Cylinder Functions] [Electronic Resource]. Available at: http://num-anal.srcc.msu.ru/lib_na/cat/cat64.htm (accessed 14.05.2018). [in Russian].

13. Amosov A.A., Dubinskii Yu.A., Kopchenova N.V. Vychislitel'nye metody dlya inzhenerov [Numerical Methods for Engineers]. Moscow, Vysshaya shkola Publ., 1994. 544 p. [in Russian]. 


\section{Сведения об авторе}

\section{About the author}

Погорелов Д.Ю., д-р физ.-мат. наук, профессор, руководитель лаборатории «Вычислительная механика», ФГБОУ ВО «БГТУ», г. Брянск, Российская Федерация

D.Yu. Pogorelov, Doctor of Physical and Mathematical Sciences, Professor, Head of Laboratory of Computational Mechanics, FSBEI HE «BSTU», Bryansk, Russian Federation

e-mail:pogorelov@umlab.ru 